\title{
REMAINDER TERM ESTIMATES IN A CONDITIONAL CENTRAL LIMIT THEOREM FOR INTEGER-VALUED RANDOM VARIABLES
}

\author{
M. P. QUINE \\ (Received 14 October 1983; revised 8 December 1983) \\ Communicated by T. C. Brown
}

\begin{abstract}
A Berry-Esseen type result is given for the conditional distribution of a weighted sum of i.i.d. integer-valued r.v.'s given that their unweighted sum equals its expectation. The examples include the case of sampling without replacement from a finite population.
\end{abstract}

1980 Mathematics subject classification (Amer. Math. Soc.): $60 \mathrm{~F} 05$.

\section{Introduction and main results}

Let $X_{1}^{\prime}, \ldots, X_{n}^{\prime}$ be independent and identically distributed integer-valued random variables with maximal span 1 . Suppose $E X_{1}^{\prime}=\mu, E\left(X_{1}^{\prime}-\mu\right)^{2}=\sigma^{2}>0$, and set $X_{k}=\left(X_{k}^{\prime}-\mu\right) / \sigma, k=1, \ldots, n, \rho=E\left|X_{1}\right|^{3}$. Let $a_{1}, \ldots, a_{n}$ be real constants satisfying $\Sigma_{k} a_{k}=0, \Sigma_{k} a_{k}^{2}=n$. We seek bounds on the quantity

$$
\Delta=\sup _{x}\left|P\left(n^{-1 / 2} \sum_{k} a_{k} X_{k} \leqslant x \mid \sum_{k} X_{k}^{\prime}=n \mu\right)-(2 \pi)^{-1 / 2} \int_{-\infty}^{x} e^{-u^{2} / 2} d u\right| ;
$$

for $\Delta$ to be well defined, of course, $n \mu$ must be integral, so that the distribution of $X_{1}^{\prime}$ itself may depend on $n$. In general our bound on $\Delta$ is a rather "unnatural" one, involving an estimate of the absolute value of the characteristic function $g(w)=E e^{i w X_{1}}$, which is provided by the following basic lemma. However in the special cases considered later, the estimate is easy to calculate and manipulate.

(C) 1985 Australian Mathematical Society 0263-6115/85\$A2.00+0.00 
Lemma 1. For any $b \in[0, \pi)$ there exists a constant $\eta_{b} \in\left(0, \frac{1}{2}\right)$ such that $|g(w)|<e^{-\eta_{b} w^{2}}, 0<|w|<(\pi+b) \sigma$.

Set $d_{n}=\left(2 \pi \sigma n^{1 / 2} P\left(\sum_{k} X_{k}^{\prime}=n \mu\right)\right)^{-1}$ and $T=n^{3 / 2}\left(\rho \sum_{k}\left|a_{k}\right|^{3}\right)^{-1}$.

TheOREM. If $\varepsilon, \delta$ and $\gamma$ are positive constants with $\varepsilon<2^{-1 / 2}, \gamma<\frac{1}{2}(\sqrt{5}-1)$, $b=2 \delta / \gamma<\pi$, then

$$
\Delta \leqslant \frac{1}{(4 \alpha-2) T}\left\{Q_{1}+Q_{2}+Q_{3}+Q_{4}+n^{-1 / 2} Q_{5}\right\},
$$

where, choosing $H$ so that

$$
\alpha=\frac{1}{\pi} \int_{-H}^{H}\left(\frac{\sin y}{y}\right)^{2} d y>\frac{1}{2},
$$

$$
\begin{aligned}
& Q_{1}=1.596 \alpha H / \delta, \\
& Q_{2}=\frac{2 d_{n}}{\pi \eta_{b}^{2}}\left\{\frac{1}{4}\left(\frac{1}{2}+\frac{\varepsilon}{1-2 \varepsilon^{2}}\right)\left(\frac{\pi}{\beta_{1}}+\frac{4}{\beta_{2}}+\frac{\pi}{\beta_{3}}\right)+\frac{1}{2 \varepsilon}\left(\frac{1}{\beta_{2}}+\frac{c_{b}}{\beta_{2}^{*}}+\frac{\pi c_{b}}{2 \beta_{3}^{*}}\right)\right\}, \\
& Q_{3}=\frac{d_{n} c_{b}}{6 \pi \eta_{b}^{2}}\left\{\frac{\pi}{2 \beta_{1}^{*}}+\frac{3}{\beta_{2}^{*}}+\frac{3 \pi}{2 \beta_{3}^{*}}+\frac{\pi^{1 / 2}}{\varepsilon \beta_{3}^{*}\left(\alpha_{2}^{*} \eta_{b}\right)^{1 / 2}}+\frac{3 \pi^{1 / 2}}{4 \beta_{1}^{*}\left(\alpha_{1} \eta_{b}\right)^{1 / 2}}\right\} \\
& Q_{4}=\frac{0.133 d_{n}}{\varepsilon \eta_{0}^{2}(1-1 / n)^{2}}, \\
& Q_{5}=\frac{0.071 d_{n} c_{b}}{\alpha_{1}^{1 / 2} \eta_{b}^{5 / 2}}\left\{\frac{2}{\beta_{2}^{*}}+\frac{3}{\beta_{3}^{*}}+\frac{4}{\alpha_{2}^{* 2}}+\frac{1.33}{\alpha_{2}^{* 5 / 2} \eta_{b}^{1 / 2} \varepsilon}\right\}+\frac{1.061 d_{n}}{\eta_{0}^{5 / 2}(1-1 / n)^{5 / 2} \varepsilon}, \\
& \alpha_{1}=1-\gamma-\gamma^{2}, \quad \alpha_{2}=1-\gamma^{2}-\gamma^{3}, \quad \alpha_{2}^{*}=\alpha_{2}-2 / n, \\
& \beta_{1}=\alpha_{1}^{3 / 2} \alpha_{2}^{1 / 2}, \quad \beta_{2}=\alpha_{1} \alpha_{2}, \quad \beta_{3}=\alpha_{1}^{1 / 2} \alpha_{2}^{3 / 2}, \\
& \beta_{1}^{*}=\alpha_{1}^{3 / 2} \alpha_{2}^{* 1 / 2}, \quad \beta_{2}^{*}=\alpha_{1} \alpha_{2}^{*}, \quad \beta_{3}^{*}=\alpha_{1}^{1 / 2} \alpha_{2}^{* 3 / 2},
\end{aligned}
$$

and

$$
c_{b}=\exp \left(2 \eta_{b} \delta^{2} / \gamma^{2}\right) .
$$

The theorem is given in this cumbersome form to facilitate computation of the bound in particular cases (see Section 2). The following corollaries may suffice in some applications.

COROLlaRY 1. There exists a constant $C$ such that $\Delta \leqslant C d_{n} / T$.

The factor $d_{n}$ may also be removed in many situations, where the distributions under consideration are "uniformly aperiodic", as the following corollary shows.

COROLlary 2. If $\eta_{0} \geqslant \eta_{0}^{*}>0$, then $\Delta \leqslant C / T$. 
Finally we remark that if $X_{1}^{\prime}$ has maximal span $\lambda>1$, then the above results can be applied to the variables $\lambda^{-1} X_{k}^{\prime}$. In the next section we look at some examples, and in Section 3 we provide proofs.

\section{Two examples}

First we show how Corollary 2 can be used in a simple example. Suppose

$$
P\left(X_{k}^{\prime}=+1\right)=P\left(X_{k}^{\prime}=-1\right)=p<\frac{1}{2}, \quad P\left(X_{k}^{\prime}=0\right)=1-2 p
$$

for $k=1,2, \ldots$ Then $g(w)$ and $\eta_{0}>0$ do not depend on $n$, and with $a_{k}=(-1)^{k}$, Corollary 2 gives

$$
\begin{aligned}
\Delta & =\sup _{x}\left|P\left(\sum_{k=1}^{2 n}(-1)^{k} X_{k}^{\prime} \leqslant 2(n p)^{1 / 2} x \mid \sum_{k=1}^{2 n} X_{k}^{\prime}=0\right)-(2 \pi)^{-1 / 2} \int_{-\infty}^{x} e^{-u^{2} / 2} d u\right| \\
& =O\left(n^{-1 / 2}\right) .
\end{aligned}
$$

For a given value of $p$, the theorem itself could be used to find a numerical constant $C_{p}$ such that $\Delta<C_{p} n^{-1 / 2}$, by methods which we will now demonstrate in a different context.

Suppose $P\left(X_{1}^{\prime}=1\right)=p, P\left(X_{1}^{\prime}=0\right)=q=1-p$. The problem is then equivalent to that of approximating the distribution of a sample of size $n p$ drawn without replacement from the finite population $\left(a_{1}, \ldots, a_{n}\right)$. Höglund (1976) has shown that the order term is $O\left(T^{-1}\right)$; we will use our theorem to show $\Delta<145 / T$. The characteristic function satisfies $|g(w)|^{2}=1+2 p q(\cos (w / \sigma)-1)$, so

$$
|g(w)| \leqslant e^{p q(\cos (w / \sigma)-1)} \leqslant \exp \left(-\eta_{b}^{*} w^{2}\right), \quad|w| \leqslant(\pi+b) \sigma,
$$

where $\eta_{b}^{*}=(1-\cos (\pi+b)) /(\pi+b)^{2}$. Bhattacharya and Ranga Rao (1976, Lemma 12.3) show that it is always the case that $\Delta \leqslant 0.5416$. So we may take $T>200$, whereby the bound (21) below (which is used in proving Corollary 2) gives $d_{n} \leqslant 0.416$. A numerical integration of (2) was performed (this can also be evaluated from one of the many tables of $\operatorname{Si}(x)$, since $\int_{0}^{x}((\sin y) / y)^{2} d y=\operatorname{Si}(2 x)$ $-\left(\sin ^{2} x\right) / x$. See for example Abramowitz and Stegun (1965, page 236) for references to tables), and the right hand side of (1) was minimized (with $Q_{5}=0$ ) over the range $0.86<H<3$; the resulting minimum of 144.4 was found at $H=2.16, \alpha=0.876, \varepsilon=0.45, \delta=0.04, \gamma=0.22$. Here $Q_{5}<84$ and it follows that $\Delta<145 / T$.

Finally, we note that other applications may be found in Holst (1979), where a corresponding central limit theorem is proved. 


\section{Proofs}

First we prove Lemma 1. Since $X_{1}$ has maximal span $\sigma^{-1}$, there exists for any $\varepsilon>0$ a number $k_{\varepsilon}>0$ such that $|g(w)|<e^{-k_{\varepsilon}}, \varepsilon<|w|<2 \pi \sigma-\varepsilon$ (Gnedenko (1963), page 297). Also, if $\varepsilon<\varepsilon_{0}=\min \left(1.5 / \rho, 2^{1 / 2}\right)$, then for $|w|<\varepsilon,|g(w)|<1$ $-\frac{1}{2} w^{2}+\frac{1}{6} \rho|w|^{3}<e^{-w^{2} / 4}$. Assume without loss of generality that $e^{-k_{\varepsilon}} \geqslant e^{-\varepsilon^{2} / 4}$. Then for $b=\pi-\sigma^{-1} \varepsilon>b_{0}=\pi-\sigma^{-1} \varepsilon_{0}$, the lemma holds with $\eta_{b}=$ $k_{\varepsilon} /(\pi+b)^{2} \sigma^{2}$. If $b_{0}>0$ then for $b<b_{0}$ the lemma holds with $\eta_{b}=\eta_{b_{0}}$.

We now turn to the proof of the theorem. Let

$$
k(t)= \begin{cases}1-\frac{|t|}{\delta T}, & |t| \leqslant \delta T \\ 0, & \text { otherwise. }\end{cases}
$$

This is the characteristic function of the probability measure with density

$$
f(x)=\frac{\delta T}{2 \pi}\left(\frac{\sin \frac{1}{2} \delta T x}{\frac{1}{2} \delta T x}\right)^{2}, \quad-\infty<x<\infty .
$$

Taking $H$ and $\alpha$ as in (2), Lemma (12.2) of Bhattacharya and Ranga Rao gives

$$
\Delta \leqslant \frac{1}{2 \alpha-1}\left[\frac{1}{2 \pi} \int_{|t| \leqslant \delta T}|t|^{-1}\left|\psi(t)-e^{-t^{2} / 2}\right| d t+\frac{2 \alpha H}{(2 \pi)^{1 / 2} \delta T}\right]
$$

where $\psi(t)=E\left\{\exp \left(i t n^{-1 / 2} \sum_{k} a_{k} X_{k} \mid \sum_{k} X_{k}^{\prime}=n \mu\right)\right\}$. It follows from Bartlett (1938) that

$$
\begin{aligned}
\psi(t)= & \left\{2 \pi P\left(\sum_{k} X_{k}^{\prime}=n \mu\right)\right\}^{-1} \\
& \times \int_{-\pi}^{\pi} E\left\{\exp \left(i x\left(\sum_{k} X_{k}^{\prime}-n \mu\right)+i t n^{-1 / 2} \sum_{k} a_{k} X_{k}\right)\right\} d x \\
= & d_{n} \int_{-\pi \sigma n^{1 / 2}}^{\pi \sigma n^{1 / 2}} \prod_{k} g_{k}(t, v) d v,
\end{aligned}
$$

where $g_{k}(t, v)=g\left(n^{-1 / 2}\left(t a_{k}+v\right)\right)$. For $t>0$,

(5) $\left|\psi(t)-e^{-t^{2} / 2}\right|=e^{-t^{2} / 2}\left|\int_{0}^{t} \frac{d}{d s} e^{s^{2} / 2} \psi(s) d s\right| \leqslant|t| e^{-t^{2} / 2} \sup _{|s| \leqslant|t|}\left|\frac{d}{d s} e^{s^{2} / 2} \psi(s)\right|$,

and similarly this inequality also obtains for $t<0$. We will use (4) and (5) to bound the integral in (3).

The next lemma is due to Höglund (1976).

LEMMA 2. Let $K_{\gamma}=\left\{k: 1 \leqslant k \leqslant n, \quad \gamma\left|a_{k}\right| \leqslant \Sigma_{j}\left|a_{j}\right|^{3} / n\right\}, \quad 0<\gamma<1$. Then $\sum_{k \in K_{y}}\left(a_{k} x+y\right)^{2} / n \geqslant \alpha_{1} x^{2}+\alpha_{2} y^{2}$, where $\alpha_{1}$ and $\alpha_{2}$ are defined in the theorem and are positive if $2 \gamma<\sqrt{5}-1$. 
Proof. Hölder's inequality gives $\sum_{k \in K_{r}} 1 \geqslant\left(1-\gamma^{3}\right) n, \Sigma_{k \in K_{r}} a_{k}^{2} \geqslant(1-\gamma) n$, and $\sum_{k \notin K_{\gamma}}\left|a_{k}\right| \leqslant \gamma^{2} n$. So

$$
\begin{aligned}
\sum_{k \in K_{\gamma}}\left(a_{k} x+y\right)^{2} / n & \geqslant(1-\gamma) x^{2}+\left(1-\gamma^{3}\right) y^{2}-2 \sum_{k \notin K_{\gamma}} a_{k} x y / n \\
& \geqslant(1-\gamma) x^{2}+\left(1-\gamma^{3}\right) y^{2}-2|x y| \gamma^{2} \\
& \geqslant\left(1-\gamma-\gamma^{2}\right) x^{2}+\left(1-\gamma^{2}-\gamma^{3}\right) y^{2}
\end{aligned}
$$

LEMMA 3. Let $\left(d_{j},-\infty<j<\infty\right)$ be constants, $d_{j}<d_{j+1}$ for all $j$; let $Z$ be a r.v. with $P\left(Z=d_{j}\right)=p_{j}$ for all $j, \Sigma_{j} p_{j}=1, E Z=\mu, \min _{j}\left(d_{j}-d_{j-1}\right)=\varepsilon>0$. Then

$$
\frac{E|Z-\mu|^{2}}{E|Z-\mu|^{3}} \leqslant \frac{2}{\varepsilon} \text {. }
$$

Proof. Let $n$ be the integer such that $d_{n} \leqslant \mu<d_{n+1}$; let $\alpha=\mu-d_{n}, \beta=d_{n+1}$ $-\mu, \delta=\alpha+\beta, \alpha^{\prime}=\alpha / \delta, \beta^{\prime}=\beta / \delta$. Then $E(Z-\mu)=0$ implies

$$
\alpha p_{n}-\beta p_{n+1}=\sum_{j<n}\left(d_{j}-\mu\right) p_{j}+\sum_{j>n+1}\left(d_{j}-\mu\right) p_{j} .
$$

Since $\alpha^{\prime}+\beta^{\prime}=1$,

$\alpha^{3} p_{n}+\beta^{\prime 3} p_{n+1}=\left(\alpha^{\prime 2}+\beta^{\prime 2}\right)\left(\alpha^{\prime 2} p_{n}+\beta^{\prime 2} p_{n+1}\right)+\alpha^{\prime} \beta^{\prime}\left(\alpha^{\prime}-\beta^{\prime}\right)\left(\alpha^{\prime} p_{n}-\beta^{\prime} p_{n+1}\right)$ so

$$
\begin{aligned}
E|Z-\mu|^{3} & =\alpha^{3} p_{n}+\beta^{3} p_{n+1}+\sum_{j \neq n, n+1}\left|d_{j}-\mu\right|^{3} p_{j} \\
\geqslant & \delta^{3}\left\{\left(\alpha^{\prime 2}+\beta^{\prime 2}\right)\left(\alpha^{\prime 2} p_{n}+\beta^{\prime 2} p_{n+1}\right)+\alpha^{\prime} \beta^{\prime}\left(\alpha^{\prime}-\beta^{\prime}\right)\left(\alpha^{\prime} p_{n}-\beta^{\prime} p_{n+1}\right)\right\} \\
& +\sum_{j \neq n, n+1}\left|d_{j}-\mu\right|^{3} p_{j} \\
\geqslant & \left(\alpha^{\prime 2}+\beta^{\prime 2}\right) \delta\left\{\alpha^{2} p_{n}+\beta^{2} p_{n+1}\right\}-\delta^{2} \alpha^{\prime} \beta^{\prime}\left(\alpha^{\prime}-\beta^{\prime}\right) \sum_{j<n}\left(\left(d_{j}-\mu\right)^{2} / \alpha\right) p_{j} \\
& +\sum_{j \neq n, n+1}\left|d_{j}-\mu\right|^{3} p_{j} \quad(\text { if } \alpha>\beta) \\
\geqslant & \frac{1}{2} \delta\left\{\alpha^{2} p_{n}+\beta^{2} p_{n+1}\right\}+\left\{\varepsilon+\alpha-\delta \beta^{\prime}\left(\alpha^{\prime}-\beta^{\prime}\right)\right\} \sum_{j<n}\left(d_{j}-\mu\right)^{2} p_{j} \\
& +(\varepsilon+\beta) \sum_{j>n+1}\left(d_{j}-\mu\right)^{2} p_{j} \\
\geqslant & \frac{1}{2} \delta\left(\alpha^{2} p_{n}+\beta^{2} p_{n+1}\right)+\left(\varepsilon+\frac{1}{2} \delta\right) \sum_{j<n}\left(d_{j}-\mu\right)^{2} p_{j}+\varepsilon \sum_{j>n+1}\left(d_{j}-\mu\right)^{2} p_{j} \\
\geqslant & \frac{1}{2} \varepsilon \sigma^{2} .
\end{aligned}
$$

A similar argument applies if $\alpha \leqslant \beta$. 
REMARK. This inequality is sharp when $\varepsilon=1$ for a r.v. $Z$ with $P(Z=0)=$ $P(Z=1)=\frac{1}{2}$.

LeMMA 4. For $|s| \leqslant \delta T,|v| \leqslant \pi \sigma n^{1 / 2}, \prod_{k} g_{k}(s, v) \mid \leqslant \exp \left(-\eta_{b}\left(\alpha_{1} s^{2}+\alpha_{2} v^{2}\right)\right)$ so long as $b=2 \delta / \gamma<\pi$.

Proof. If $k \in K_{r}$, then

$$
n^{-1 / 2}\left|s a_{k}\right| \leqslant \delta / \rho \gamma \leqslant \delta / \gamma,
$$

so $n^{-1 / 2}\left|s a_{k}+v\right| \leqslant(2 \delta / \gamma+\pi)$ a from Lemma 3. So Lemma 1 gives

$$
\left|\prod_{k} g_{k}(s, v)\right| \leqslant\left|\prod_{k \in K_{\gamma}} g_{k}(s, v)\right| \leqslant \exp \left(-\eta_{b} \sum_{k \in K_{\gamma}}\left(s a_{k}+v\right)^{2} / n\right)
$$

and the result follows from Lemma 2.

We can now consider the integral in (3). Let

$$
I_{1}=\int_{-\varepsilon U}^{\varepsilon U}|t|^{-1}\left|\psi(t)-e^{-t^{2} / 2}\right| d t \leqslant d_{n} \int_{-\varepsilon U}^{\varepsilon U} e^{-t^{2} / 2}\left(J_{11}(t)+J_{12}(t)\right) d t
$$

using (4) and (5), where $0<\varepsilon<2^{-1 / 2}, U=n^{1 / 2} / \max _{k}\left|a_{k}\right|$, and

$$
\begin{gathered}
J_{11}(t)=\int_{-\varepsilon n^{1 / 2}}^{\varepsilon n^{1 / 2}} \sup _{|s| \leqslant|t|}\left|\frac{d}{d s} e^{s^{2} / 2} \prod_{k} g_{k}(s, v)\right| d v, \\
J_{12}(t)=\int_{\varepsilon n^{1 / 2}<|v|<\pi \sigma n^{1 / 2}} \sup _{|s| \leqslant|t|}\left|\frac{d}{d s} e^{s^{2} / 2} \prod_{k} g_{k}(s, v)\right| d v .
\end{gathered}
$$

Here and in the sequel we assume that $b=2 \delta / \gamma<\pi$ as in Lemma 4.

LemMa 5. For $|s| \leqslant \min (\varepsilon U, \delta T),|v| \leqslant \varepsilon n^{1 / 2}$,

$$
\begin{aligned}
& \left|\frac{d}{d s} e^{s^{2} / 2} \prod_{k} g_{k}(s, v)\right| \\
& \quad \leqslant\left(\frac{1}{2} \rho+\frac{\varepsilon}{1-2 \varepsilon^{2}}\right) n^{-3 / 2} \sum_{k}\left|a_{k}\right|\left(s a_{k}+v\right)^{2} \exp \left(\frac{1}{2} s^{2}-\eta_{b}\left(\alpha_{1} s^{2}+\alpha_{2} v^{2}\right)\right) .
\end{aligned}
$$

Proof. First, note that

(8) $\left|g_{k}(s, v)-1\right|=\left|E\left\{\exp \left(i X_{k}\left(s a_{k}+v\right) n^{-1 / 2}\right)\right\}-1\right| \leqslant \frac{1}{2}\left(s a_{k}+v\right)^{2} / n$

$$
\leqslant\left(\left(s a_{k}\right)^{2}+v^{2}\right) / n \leqslant 2 \varepsilon^{2} \text {. }
$$

Let $h(s, v)=\sum_{k} \log g_{k}(s, v)+\frac{1}{2} s^{2}+\frac{1}{2} v^{2}$. Then

(10)

$$
\begin{aligned}
\left|\frac{d}{d s} h(s, v)\right| & \leqslant\left|\sum_{k} \frac{d}{d s} g_{k}(s, v)+s\right|+\left|\sum_{k} g_{k}^{-1}(s, v)\left(1-g_{k}(s, v)\right) \frac{d}{d s} g_{k}(s, v)\right| \\
& =A_{1}+A_{2}
\end{aligned}
$$


say, and

$$
\text { (11) } \begin{aligned}
A_{1} & =\left|\sum_{k} E\left\{i X_{k} a_{k} n^{-1 / 2}\left(e^{i X_{k}\left(s a_{k}+v\right)} n^{-1 / 2}-1-i X_{k}\left(s a_{k}+v\right) n^{-1 / 2}\right)\right\}\right| \\
& \leqslant \frac{1}{2} \sum_{k}\left(s a_{k}+v\right)^{2}\left|a_{k}\right| \rho n^{-3 / 2} .
\end{aligned}
$$

From (8) and (9),

(12)

$$
\begin{aligned}
A_{2} & \leqslant\left(1-2 \varepsilon^{2}\right)^{-1} \sum_{k}\left|1-g_{k}(s, v)\right| \cdot\left|\frac{d}{d s} g_{k}(s, v)\right| \\
& \leqslant \varepsilon\left(1-2 \varepsilon^{2}\right)^{-1} n^{-1 / 2} \sum_{k}\left|s a_{k}+v\right| \cdot\left|E\left\{i a_{k} X_{k} n^{-1 / 2}\left(e^{i X_{k}\left(s a_{k}+v\right) n^{-1 / 2}}-1\right)\right\}\right| \\
& \leqslant \varepsilon\left(1-2 \varepsilon^{2}\right)^{-1} n^{-3 / 2} \sum_{k}\left(s a_{k}+v\right)^{2}\left|a_{k}\right| .
\end{aligned}
$$

Since

$$
\begin{aligned}
\left|\frac{d}{d s} e^{s^{2} / 2} \prod_{k} g_{k}(s, v)\right| & =\left|\frac{d}{d s} e^{h(s, v)-v^{2} / 2}\right| \leqslant\left|\frac{d}{d s} h(s, v)\right| \cdot\left|e^{h(s, v)-v^{2} / 2}\right| \\
& =e^{s^{2} / 2}\left|\frac{d}{d s} h(s, v)\right| \cdot\left|\prod_{k} g_{k}(s, v)\right|,
\end{aligned}
$$

the lemma follows from (10)-(12) and Lemma 4.

Lemma 6. For $|s| \leqslant \min (\varepsilon U, \delta T), \varepsilon n^{1 / 2}<|v| \leqslant \pi \sigma n^{1 / 2}$,

$$
\begin{aligned}
\left|\frac{d}{d s} e^{s^{2} / 2} \prod_{k} g_{k}(s, v)\right| \leqslant & |s| \exp \left(\frac{1}{2} s^{2}-\eta_{b}\left(\alpha_{1} s^{2}+\alpha_{2} v^{2}\right)\right) \\
& +(|s|+|v|) c_{b} \exp \left(\frac{1}{2} s^{2}-\eta_{b}\left(\alpha_{1} s^{2}+\left(\alpha_{2}-2 / n\right) v^{2}\right)\right) .
\end{aligned}
$$

Proof. We have

$$
\begin{aligned}
& \left|\frac{d}{d s} e^{s^{2} / 2} \prod_{k} g_{k}(s, v)\right| \leqslant|s| e^{s^{2} / 2}\left|\prod_{k} g_{k}(s, v)\right| \\
& +e^{s^{2} / 2}\left|\sum_{j} \frac{d}{d s} g_{j}(s, v) \prod_{k \neq j} g_{k}(s, v)\right|=B_{1}+B_{2},
\end{aligned}
$$


say. Lemma 4 gives $B_{1} \leqslant|s| \exp \left(\frac{1}{2} s^{2}-\eta_{b}\left(\alpha_{1} s^{2}+\alpha_{2} v^{2}\right)\right)$, and as in the derivation of (12) we get

$$
\begin{aligned}
B_{2} & \leqslant n^{-1} e^{s^{2} / 2} \sum_{j}\left|a_{j}\right| \cdot\left|s a_{j}+v\right| \cdot\left|\prod_{k \neq j, k \in K_{\gamma}} g_{k}(s, v)\right| \\
& \leqslant n^{-1} e^{s^{2} / 2} \sum_{j}\left|a_{j}\right| \cdot\left|s a_{j}+v\right| \exp \left\{-\eta_{b}\left(\alpha_{1} s^{2}+\alpha_{2} v^{2}\right)+\eta_{b} \max _{k \in K_{\gamma}}\left(s a_{k}+v\right)^{2} / n\right\} ;
\end{aligned}
$$

the lemma follows from (6), since $\rho \geqslant 1$.

The assumption $\gamma<\frac{1}{2}(\sqrt{5}-1)$ ensures $\alpha_{1}, \alpha_{2}>0$. So using Lemmas 5 and 6 on (7) gives

$$
\begin{aligned}
I_{1} \leqslant & n^{-3 / 2} d_{n}\left(\frac{1}{2} \rho+\frac{\varepsilon}{1-2 \varepsilon^{2}}\right) \int_{-\varepsilon U^{\varepsilon}}^{\varepsilon U} \int_{-\varepsilon n^{1 / 2}}^{\varepsilon n^{1 / 2}} \sum_{k}\left|a_{k}\right|\left(t a_{k}+v\right)^{2} \\
& +d_{n} \int_{-\varepsilon U}^{\varepsilon U} \int_{\varepsilon n^{1 / 2}<|v|<\pi \sigma n^{1 / 2}} t \exp \left(-\eta_{b}\left(\alpha_{1} t^{2}+\alpha_{2} v^{2}\right)\right) d v d t \\
& +d_{n} \int_{-\varepsilon U}^{\varepsilon U} \int_{\varepsilon n^{1 / 2}<|v|<\pi \sigma n^{1 / 2}}(|t|+|v|) c_{b} \exp \left(-\eta_{b}\left(\alpha_{1} t^{2}+\left(\alpha_{2} t^{2}+\alpha_{2} v^{2}\right)\right) d v d t\right. \\
\leqslant & \frac{1}{2} d_{n} n^{-3 / 2}\left(\frac{1}{2} \rho+\frac{\varepsilon}{1-2 \varepsilon^{2}}\right) \eta_{b}^{-2} \sum_{k}\left(\frac{\pi\left|a_{k}\right|^{3}}{\beta_{1}}+\frac{4 a_{k}^{2}}{\beta_{2}}+\frac{\pi\left|a_{k}\right|}{\beta_{3}}\right) \\
& +\frac{d_{n} n^{-1 / 2}}{\varepsilon \eta_{b}^{2}}\left(\frac{1}{\beta_{2}}+\frac{c_{b}}{\alpha_{1} \alpha_{2}^{*}}+\frac{\pi c_{b}}{2 \alpha_{1}^{1 / 2} \alpha_{2}^{* 3 / 2}}\right) .
\end{aligned}
$$

If $\varepsilon U<\delta T$ we have also to consider the integral

$$
\text { (14) } \begin{aligned}
I_{2} & =\int_{\varepsilon U<|t|<\delta T}|t|^{-1}\left|\psi(t)-e^{-t^{2} / 2}\right| d t \\
& =\int_{\varepsilon U<|t|<\delta T}|t|^{-1}\left|\psi(t)-\psi(0) e^{-t^{2} / 2}\right| d t \\
& =d_{n} \int_{\varepsilon U<|t|<\delta T}|t|^{-1}\left|\int_{-\pi \sigma n}^{\pi \sigma n^{1 / 2}}\left(\prod_{k} g_{k}(t, v)-e^{-t^{2} / 2} \prod_{k} g_{k}(0, v)\right) d v\right| d t \\
& \leqslant d_{n} \int_{\varepsilon U<|t|<\delta T}|t|^{-1}\left(J_{21}(t)+J_{22}(t)\right) d t,
\end{aligned}
$$


where

$$
J_{21}(t)=\int_{-\pi \sigma n^{1 / 2}}^{\pi \sigma n^{1 / 2}}\left|\prod_{k} g_{k}(t, v)-e^{-\left(t^{2}+v^{2}\right) / 2}\right| d v
$$

and

$$
J_{22}(t)=\int_{-\pi \sigma n^{1 / 2}}^{\pi \sigma n^{1 / 2}} e^{-t^{2} / 2}\left|g^{n}\left(v n^{-1 / 2}\right)-e^{-v^{2} / 2}\right| d v
$$

The integrand in $J_{21}(t)$ is

$$
\begin{aligned}
& \left|\prod_{k} g_{k}(t, v)-\prod_{k} e^{-\left(t a_{k}+v\right)^{2} /(2 n)}\right| \\
& \quad \leqslant \sum_{j=1}^{n}\left|\prod_{k=1}^{j-1} g_{k}(t, v)\left\{g_{j}(t, v)-e^{-\left(t a_{j}+v\right)^{2} /(2 n)}\right\} \prod_{k=j+1}^{n} e^{-\left(t a_{k}+v\right)^{2} /(2 n)}\right| .
\end{aligned}
$$

We have

$$
\begin{aligned}
\mid g_{j}(t, v)-e^{-\left(t a_{j}+v\right)^{2} /(2 n) \mid \leqslant} & \left|g_{j}(t, v)-1+\frac{1}{2}\left(t a_{j}+v\right)^{2} / n\right| \\
& +\left|e^{-\left(t a_{j}+v\right)^{2} /(2 n)}-1+\frac{1}{2}\left(t a_{j}+v\right)^{2} / n\right| \\
\leqslant & \frac{1}{6} \rho\left|t a_{j}+v\right|^{3} n^{-3 / 2}+\frac{1}{8}\left(t a_{j}+v\right)^{4} n^{-2} .
\end{aligned}
$$

For $k \in K_{j}, n^{-1 / 2}\left|t a_{k}+v\right| \leqslant(2 \delta / \gamma+\pi) \sigma$ as in the proof of Lemma 4 , so with $b$ as before,

$$
\begin{aligned}
\left|\prod_{k=1}^{j-1} g_{k}(t, v) \prod_{k=j+1}^{n} e^{-\left(t a_{k}+v\right)^{2} /(2 n)}\right| \\
\quad \leqslant \exp \left\{-\eta_{b} \sum_{k<j, k \in K_{\gamma}}\left(t a_{k}+v\right)^{2} / n-\frac{1}{2} \sum_{k>j}\left(t a_{k}+v\right)^{2} / n\right\} \\
\quad \leqslant \exp \left\{-\eta_{b} \sum_{k \in K_{\gamma}}\left(t a_{k}+v\right)^{2} / n+\eta_{b} \max _{j \in K_{\gamma}}\left(t a_{j}+v\right)^{2} / n\right\} \\
\leqslant c_{b} \exp \left(-\eta_{b}\left(\alpha_{1} t^{2}+\alpha_{2}^{*} v^{2}\right)\right)
\end{aligned}
$$

from (6) and Lemma 2. From (15)-(17),

(18)

$$
J_{21}(t) \leqslant \int_{-\pi \sigma n^{1 / 2}}^{\pi \sigma n^{1 / 2}} \sum_{j}\left(\frac{1}{6} \rho\left|t a_{j}+v\right|^{3} n^{-3 / 2}+\frac{1}{8}\left(t a_{j}+v\right)^{4} n^{-2}\right) c_{b} e^{-\eta_{b}\left(\alpha_{1} t^{2}+\alpha_{2}^{*} v^{2}\right)} d v
$$


Now consider $J_{22}(t)$. Since $\left|v n^{-1 / 2}\right|<\pi \sigma,(1)$ gives $\left|g\left(v n^{-1 / 2}\right)\right|<e^{-\eta_{0} v^{2} / n}$, and setting $t=0$ in (16) gives

$$
\left|g\left(v n^{-1 / 2}\right)-e^{-v^{2} /(2 n)}\right| \leqslant \frac{1}{6} \rho|v|^{3} n^{-3 / 2}+\frac{1}{8} v^{4} n^{-2} .
$$

So since $\left|\alpha^{n}-\beta^{n}\right| \leqslant n|\alpha-\beta|(\max (|\alpha|,|\beta|))^{n-1}$,

$$
\begin{aligned}
J_{22}(t) & \leqslant n e^{-t^{2} / 2} \int_{-\pi \sigma n^{1 / 2}}^{\pi \sigma n^{1 / 2}}\left(\frac{1}{6} \rho|v|^{3} n^{-3 / 2}+\frac{1}{8} v^{4} n^{-2}\right) e^{-\eta_{0}(1-1 / n) v^{2}} d v \\
& \leqslant n e^{-t^{2} / 2}\left(\frac{\rho n^{-3 / 2}}{6 \eta_{0}^{2}(1-1 / n)^{2}}+\frac{3 \pi^{1 / 2} n^{-2}}{4 \eta_{0}^{5 / 2}(1-1 / n)^{5 / 2}}\right) .
\end{aligned}
$$

Combining (14) with (18) and (19) gives

(20)

$$
\begin{aligned}
I_{2} \leqslant & \frac{1}{6} d_{n} \rho n^{-3 / 2} c_{b} \eta_{b}^{-2} \sum_{j}\left(\frac{\left|a_{j}\right|^{3} \pi}{2 \beta_{1}^{*}}+\frac{3 a_{j}^{2}}{\beta_{2}^{*}}+\frac{3 \pi\left|a_{j}\right|}{2 \beta_{3}^{*}}+\frac{\pi^{1 / 2}}{\varepsilon U \alpha_{1}^{1 / 2} \alpha_{2}^{* 2} \eta_{b}^{1 / 2}}\right) \\
& +\frac{1}{8} d_{n} n^{-2} c_{b} \eta_{b}^{-5 / 2} \frac{\pi^{1 / 2}}{\alpha_{1}^{1 / 2}} \sum_{j}\left(\frac{a_{j}^{4}}{\beta_{1}^{*}}+\frac{2\left|a_{j}\right|^{3}}{\beta_{2}^{*}}+\frac{3 a_{j}^{2}}{\beta_{3}^{*}}+\frac{4\left|a_{j}\right|}{\alpha_{2}^{* 2}}+\frac{3 \pi^{1 / 2}}{4 \varepsilon U \alpha_{2}^{* 5 / 2} \eta_{b}^{1 / 2}}\right) \\
& +\frac{n^{-1 / 2} \rho d_{n}(2 \pi)^{1 / 2}}{6 \eta_{0}^{2}(1-1 / n)^{2} \varepsilon U}+\frac{n^{-1} d_{n} 3 \pi 2^{1 / 2}}{4 \eta_{0}^{5 / 2}(1-1 / n)^{5 / 2} \varepsilon U} .
\end{aligned}
$$

Using (3), (13) and (20) and the inequalities $\rho \geqslant 1, \sum\left|a_{k}\right| \leqslant \sum a_{k}^{2} \leqslant \Sigma\left|a_{k}\right|^{3}$, $T \leqslant n^{1 / 2}, U \geqslant 1, \max _{k}\left|a_{k}\right| \leqslant n^{1 / 2}$, we obtain the bound given in the theorem.

Proof of COROLlaries. Corollary 1 is obtained simply by choosing specific values for $\alpha, \varepsilon, \delta$ and $\gamma$; for example $\alpha=\frac{3}{4}, \varepsilon=\gamma=\delta=\frac{1}{2}$. Corollary 2 is proved as follows. Setting $t=0$ in (4) gives

$$
d_{n}^{-1}=\int_{-\pi \sigma n^{1 / 2}}^{\pi \sigma n^{1 / 2}} g^{n}\left(v n^{-1 / 2}\right) d v
$$

so

$$
\begin{aligned}
\left|d_{n}^{-1}-(2 \pi)^{1 / 2}\right| & \leqslant \int_{-\pi \sigma n^{1 / 2}}^{\pi \sigma n^{1 / 2}}\left|g^{n}\left(v n^{-1 / 2}\right)-e^{-v^{2} / 2}\right| d v+\int_{|v|>\pi \sigma n^{1 / 2}} e^{-v^{2} / 2} d v \\
& =K_{1}+K_{2},
\end{aligned}
$$

say. $K_{1}$ is just $J_{22}(0)$, which from (19) is bounded for $n \geqslant 2$ by $2 /\left(3 \eta_{0}^{2} T\right)+$ $7.52 /\left(\eta_{0}^{5 / 2} n\right)$, whilst $K_{2} \leqslant 2 /\left(\pi \sigma n^{1 / 2}\right)<1.3 / T$ from Lemma 3. So if $\eta_{0} \geqslant \eta_{0}^{*}>0$, then for $T>T_{0}$,

$$
d_{n} \leqslant\left[(2 \pi)^{1 / 2}-K_{1}-K_{2}\right]^{-1} \leqslant\left[(2 \pi)^{1 / 2}-\frac{2}{3 \eta_{0}^{* 2} T_{0}}-\frac{7.52}{\eta_{0}^{* 5 / 2} T_{0}^{2}}-\frac{1.3}{T_{0}}\right]^{-1},
$$

and Corollary 2 follows on choosing $\alpha, \varepsilon, \delta$ and $\gamma$ as before. 


\section{Acknowledgements}

I am grateful to Allan Gut for his careful reading of an early draft, to Svante Janson for programming assistance and to Lars Holst for enabling me to visit Uppsala University, where this work was carried out. I thank the referee for some useful comments.

\section{References}

M. Abramowitz and I. A. Stegun (1965), Handbook of mathematical functions (Dover, New York).

M. S. Bartlett (1938), 'The characteristic function of a conditional statistic', J. London Math. Soc. 13, 62-67.

R. N. Bhattacharya and R. Ranga Rao (1976), Normal approximation and asymptotic expansions (Wiley, New York).

B. V. Gnedenko (1962), The theory of probability (Chelsea, New York).

T. Höglund (1976), 'Sampling from a finite population: a remainder term estimate', Studia Sci. Math. Hungar. 11, 69-74.

L. Holst (1979), 'Two conditional limit theorems with applications', Ann. Statist. 1, 551-557.

Department of Mathematical Statistics

University of Sydney

Sydney, N.S.W. 2006

Australia 